\title{
State of Charge Estimation of Parallel Connected Battery Cells via Descriptor System Theory*
}

\author{
Dong Zhang ${ }^{1}$, Luis D. Couto ${ }^{2}$, Sebastien Benjamin ${ }^{3}$, Wente Zeng ${ }^{4}$, Daniel F. Coutinho ${ }^{5}$ and Scott J. Moura ${ }^{1}$
}

\begin{abstract}
This manuscript presents an algorithm for individual Lithium-ion (Li-ion) battery cell state of charge (SOC) estimation when multiple cells are connected in parallel, using only terminal voltage and total current measurements. For battery packs consisting of thousands of cells, it is desirable to estimate individual SOCs by only monitoring the total current in order to reduce sensing cost. Mathematically, series connected cells yield dynamics given by ordinary differential equations under classical full voltage sensing. In contrast, parallel connected cells are evidently more challenging because the dynamics are governed by a nonlinear descriptor system, including differential equations and algebraic equations arising from voltage and current balance across cells. This paper designs and analyzes an observer with linear output error injection, where the individual cell SOCs and local currents are locally observable from the total current and voltage measurements. The asymptotic convergence of differential and algebraic states is established by considering local Lipschitz continuity property of system nonlinearities. Simulation results on $\mathrm{LiNiMnCoO}_{2} / \mathrm{Graphite} \mathrm{NMC}$ ) cells illustrate convergence for SOCs, local currents, and terminal voltage.
\end{abstract}

\section{INTRODUCTION}

Lithium-ion (Li-ion) batteries have emerged as one of the most prominent energy storage devices for large-scale energy applications, e.g., hybrid electric vehicle (HEV), battery electric vehicles (BEV) and smart grids, due to their high energy and power density, low self-discharge and long lifetime [1]. A battery pack system generally consists of hundreds or thousands of single cells connected in parallel and series connections in order to fulfill the requirements of high-energy and high-power applications [2]. It is wellknown that Li-ion cells are sensitive to overcharge and over-discharge [3]. An accurate estimation of the internal states, including state of charge (SOC), enables a battery management system (BMS) to prolong battery service life by ensuring individual cells within a pack do not overcharge or over-discharge.

\footnotetext{
*This work is sponsored by Total S.A. and Saft Batteries.

${ }^{1}$ Dong Zhang and Scott J. Moura is with Department of Civil and Environmental Engineering, University of California, Berkeley, California, 94720, USA. \{dongzhr, smoura\} aberkeley. edu

${ }^{2}$ Luis D. Couto is with Department of Control Engineering and System Analysis, Université Libre de Bruxelles, B-1050 Brussels, Belgium. lcoutome@ulb.ac.be

${ }^{3}$ Sebastien Benjamin is with Saft Batteries, Total S.A.. Sebastien. BENJAMINQsaftbatteries.com

${ }^{4}$ Wente Zeng is with Total S.A.. wente. zengetotal.com

${ }^{5}$ Daniel F. Coutinho is with Department of Automation and Systems, UFSC, PO BOX 476, 88040-900, Florianópolis, SC, Brazil. daniel. coutinhodufsc.br
}

Battery pack system modeling can be divided into three categories. The first approach treats the entire pack as one lumped single cell [4]. However, the internal states of individual cells within the pack are often different, due to parameter heterogeneity. Therefore, some cells are more prone to violate safety-critical constraints than others, which cannot be resolved from the lumped single cell approach. The second modeling approach also relies on a single cell model, but it focuses on a set of specific in-pack cells the weakest and the strongest ones, as representatives of the pack dynamics [2], [5]. The last modeling approach is based on the interconnection of single cell models [6], [7]. This approach benefits from high fidelity cell-by-cell resolution, but it suffers from high real-time computational burden. To counteract this computational challenge, most of these approaches resort to equivalent circuit models (ECM), which have a low complexity when compared to more sophisticated electrochemical models.

The state estimation problem for series arrangements of battery cells has been studied previously [3], [8], whereas the estimation for cells in parallel has been overlooked for multiple reasons. First, cells in parallel are widely considered to behave as one single cell. However, an implicit assumption behind this reasoning is that the applied current is evenly split amongst the cells in parallel. This is hardly true due to cell heterogeneity, such as non-uniform parameter values and temperatures [9]. Secondly, the estimation problem for cells in series is arguably easier to solve than the parallel counterpart. For cells in series, the current is equal for each cell and it can be practically measured. In the parallel case, each cell's local current is unknown and determined by algebraic constraints. Due to sensing limitations, only the total current can be measured. Therefore, the parallel configuration turns out to be a differential algebraic equation (DAE) system that requires non-trivial estimation theories.

A DAE system, a.k.a. a descriptor system, involving both differential and algebraic equations, generalizes ordinary differential (normal) systems [10]. The state observer design for linear descriptor systems is a rich research topic [10], [11], [12]. In contrast, state observers for nonlinear descriptor systems is less prolific. Some relevant contributions encompass a local asymptotic state observer [13], looking at the system as differential equations on a restricted manifold [14], and an index-1 DAE observer [15]. Other works consider the case of Lipschitz nonlinearities [16], which have served as a basis for Lyapunov-based observer design using the 
linearized system [17], and LMI approaches producing state observers in singular [18] and non-singular [19] forms. Another Lipschitz system was considered in [20], where the temporal separation between slow and fast dynamics was exploited to design a robust state observer. Nonlinear descriptor systems have also been estimated through moving horizon approaches [21] and Kalman filters [22].

In light of the aforementioned literature, the contributions of this paper are threefold:

1) propose a novel framework for modeling Li-ion battery cells in parallel as a nonlinear descriptor system;

2) conduct observability analysis for this system;

3) design a Lyapunov-based asymptotic state observer for both differential and algebraic state estimation, using only voltage and total current measurements.

The reminder of this paper is organized as follows. Section II introduces the modeling framework for parallel cells. Section III motivates the problem of observer design with cell heterogeneity. Section IV provides the local observability analysis for the nonlinear descriptor system. Section V discusses the state observer design and its convergence analysis. Finally, the effectiveness of the proposed approach is illustrated in Section VI via numerical simulations. Conclusions are drawn in Section VII.

Notation. Throughout the manuscript, the symbols $I_{p \times q}$ and $\mathbf{0}_{p \times q}$ denote the identity matrix and the zero matrix with dimension $p \times q$, respectively. The inner product between $x, y \in \mathbb{R}^{n}$ is given by $\langle x, y\rangle=\sum_{i=1}^{n} x_{i} y_{i}$.

\section{Parallel Battery Model Formulation}

This section first reviews an ECM for a single battery cell, which is then electrically interconnected with other single cell models to form a parallel arrangement of cells.

\section{A. Single Battery Cell}

The ECM for a single cell $k$, consisting of an open circuit voltage $(\mathrm{OCV})$ in series connection with an ohmic resistance and an $R-C$ pair in parallel, is described by

$$
\begin{aligned}
& \dot{x}_{k}(t)=\bar{A}_{k} x_{k}(t)+\bar{B}_{k} u_{k}(t), \\
& y_{k}(t)=h_{k}\left(x_{k}(t)\right)+\bar{D}_{k} u_{k}(t),
\end{aligned}
$$

where $x_{k}=\left[\begin{array}{ll}z_{k} & V_{c, k}\end{array}\right]^{\top} \in \mathbb{R}^{2}$ is the state vector for $k$-th battery cell in the parallel connection, with $z_{k}$ as the SOC and $V_{c, k}$ as the voltage of the $R_{2, k}-C_{k}$ pair. Input $u_{k}=I_{k}$ is the current. Further, $\bar{A}_{k} \in \mathbb{R}^{2 \times 2}$ and $\bar{B}_{k} \in \mathbb{R}^{2 \times 1}$ are given by

$$
\bar{A}_{k}=\left[\begin{array}{cc}
0 & 0 \\
0 & -\frac{1}{R_{2, k} C_{k}}
\end{array}\right], \quad \bar{B}_{k}=\left[\begin{array}{c}
\frac{1}{Q_{k}} \\
\frac{1}{C_{k}}
\end{array}\right],
$$

where $Q_{k}$ represents the capacity of cell $k$. The output equation (2) for the $k$-th cell provides the voltage response

$$
h_{k}\left(x_{k}\right)=O C V\left(z_{k}\right)+V_{c, k}, \quad \bar{D}_{k}=R_{1, k},
$$

where $y_{k} \in \mathbb{R}$ is the battery terminal voltage. Function $h_{k}: \mathbb{R}^{2} \rightarrow \mathbb{R}$ consists of the open circuit voltage as a function of SOC denoted as $O C V\left(z_{k}\right)$, voltage across the $R_{2, k}-C_{k}$ pair $V_{c, k}$, and voltage response from an ohmic resistance $R_{1, k}$.

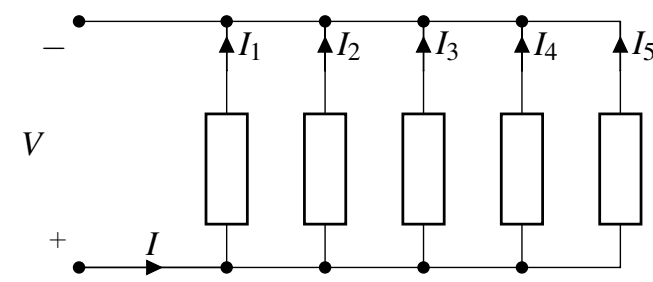

Fig. 1. Parallel connection of five battery cells.

\section{B. Parallel Arrangement of Battery Cells}

For a block of $n$ cells in parallel, in order to reduce sensing effort, we assume only the total current and voltage for the block is measured. Fig. 1 depicts a parallel connection of $n=5$ cells. Electrically, Kirchhoff's voltage law constrains cells in a parallel connection to have equal terminal voltage. Kirchhoff's current law indicates that the overall current is equal to the summation of cell local currents. Mathematically, we have the following nonlinear algebraic constraints, according to Kirchhoff's voltage law:

$$
\begin{aligned}
O C V\left(z_{i}\right)+V_{c, i}+R_{1, i} I_{i}= & O C V\left(z_{j}\right)+V_{c, j}+R_{1, j} I_{j}, \\
& \forall i, j \in\{1,2, \cdots, n\}, i \neq j .
\end{aligned}
$$

Similarly, Kirchhoff's current law poses the following linear algebraic constraint with respect to cell local currents,

$$
\sum_{k=1}^{n} I_{k}(t)=I(t)
$$

where $I(t)$ is the total current applied to the parallel battery system. Equation (5) imposes $(n-1)$ nonlinear algebraic constraints, whereas (6) imposes 1 linear algebraic constraint. In this manuscript, it is assumed that all cells have different electrical model parameters. In addition, when only the total current is measured, the local cell currents are unknown. Hence, the system of differential-algebraic equations must be solved such that the algebraic equations (5) and (6) are fulfilled for all $t$. These equations can be re-written compactly by augmenting the local currents to the differential state vector

$$
\begin{aligned}
E \dot{w}(t) & =A w(t)+\theta(w(t)), \\
y(t) & =H w(t)+\phi(w(t)),
\end{aligned}
$$

where $w=\left[\begin{array}{ll}x & u\end{array}\right]^{\top} \in \mathbb{R}^{3 n}$ with

$$
x=\left[\begin{array}{c}
x_{1} \\
\vdots \\
x_{n}
\end{array}\right] \in \mathbb{R}^{2 n}, \quad u=\left[\begin{array}{c}
I_{1} \\
\vdots \\
I_{n}
\end{array}\right] \in \mathbb{R}^{n}, \quad y=\left[\begin{array}{c}
y_{1} \\
\vdots \\
y_{n}
\end{array}\right] \in \mathbb{R}^{n} .
$$

Equation (7) encodes both system dynamical equations and algebraic equations, and $E$ is a singular matrix of the form

$$
E=\left[\begin{array}{cc}
I_{2 n \times 2 n} & \mathbf{0}_{2 n \times n} \\
\mathbf{0}_{n \times 2 n} & \mathbf{0}_{n \times n}
\end{array}\right] \in \mathbb{R}^{3 n \times 3 n},
$$

Matrix $A$ accounts for the linear part of the system equations

$$
A=\left[\begin{array}{ll}
A_{11} & A_{12} \\
A_{21} & A_{22}
\end{array}\right] \in \mathbb{R}^{3 n \times 3 n},
$$


where

$$
\begin{aligned}
A_{11}= & \operatorname{diag}\left(\bar{A}_{1}, \bar{A}_{2}, \cdots, \bar{A}_{n}\right), A_{12}=\operatorname{diag}\left(\bar{B}_{1}, \bar{B}_{2}, \cdots, \bar{B}_{n}\right), \\
A_{21}= & {\left[\begin{array}{cccccc}
0 & 1 & S & 0 & \cdots & 0 \\
0 & 1 & 0 & S & \cdots & 0 \\
\vdots & \vdots & \vdots & \vdots & \ddots & \vdots \\
0 & 1 & 0 & 0 & \cdots & S \\
0 & 0 & 0 & 0 & \cdots & 0
\end{array}\right] \in \mathbb{R}^{n \times 2 n}, \quad S=\left[\begin{array}{ll}
0 & -1
\end{array}\right], } \\
A_{22}= & {\left[\begin{array}{ccccc}
R_{1,1} & -R_{1,2} & 0 & \cdots & 0 \\
R_{1,1} & 0 & -R_{1,3} & \cdots & 0 \\
\vdots & \vdots & \vdots & \ddots & \vdots \\
R_{1,1} & 0 & 0 & \cdots & -R_{1, n} \\
1 & 1 & 1 & \cdots & 1
\end{array}\right] \in \mathbb{R}^{n \times n} . }
\end{aligned}
$$

Notice that matrix $A_{22}$ is full rank, i.e. the linear part of the descriptor model is regular and impulsive free [10].

Function $\theta(w)$ constitutes the nonlinear portion in the system equations from the voltage algebraic constraints (5):

$$
\theta(w)=\left[\begin{array}{c}
\theta_{x}(w) \\
\theta_{u}(w)
\end{array}\right]=\left[\begin{array}{c}
\mathbf{0}_{2 n \times 1} \\
O C V\left(z_{1}\right)-\operatorname{OCV}\left(z_{2}\right) \\
\vdots \\
\operatorname{OCV}\left(z_{1}\right)-\operatorname{OCV}\left(z_{n}\right) \\
-I(t)
\end{array}\right] \in \mathbb{R}^{3 n}
$$

where $\theta_{x} \in \mathbb{R}^{2 n}$ represents nonlinearity in the dynamical equations and corresponds to row 1 through $2 n$ of $\theta(w)$, and $\theta_{u} \in \mathbb{R}^{n}$ appears in the algebraic equations and corresponds to row $(2 n+1)$ through $3 n$ of $\theta(w)$. The output map (8) models the terminal voltage of each battery cell, with

$$
H=\left[\begin{array}{ll}
H_{x} & H_{u}
\end{array}\right], \quad \phi(w)=\left[\begin{array}{lll}
O C V\left(z_{1}\right) & \cdots & O C V\left(z_{n}\right)
\end{array}\right]^{\top}
$$

where

$$
\begin{aligned}
& H_{x}=\operatorname{diag}(-S,-S, \cdots,-S) \in \mathbb{R}^{n \times 2 n}, \\
& H_{u}=\operatorname{diag}\left(R_{1,1}, R_{1,2}, \cdots, R_{1, n}\right) \in \mathbb{R}^{n \times n} .
\end{aligned}
$$

The model introduced above will be used in the analysis and designs in the subsequent sections.

\section{Motivation}

In this section, we demonstrate heterogeneity for two cells in parallel via an open-loop simulation. We study a configuration of two $\mathrm{LiNiMnCoO}_{2} /$ Graphite (NMC) cells with 2.8 Ah nominal capacity in parallel. The heterogeneity arises from differences in model parameters, and in SOC initialization. A transient electric vehicle-like charge/discharge cycle generated from urban dynamometer driving schedule (UDDS) is applied. The total current is plotted in Fig. 2(a).

Two cases are examined here. In the first case, the cells are initialized at the same SOC, but they differ in model parameters. Since Cell 2 has higher resistances, its local current is smaller in magnitude relative to local current of Cell 1, as shown in Fig. 2(b) and (c). In the second case, illustrated in Fig. 2(d) and (e), the cells are initially different in both SOC and model parameters. It can be observed that
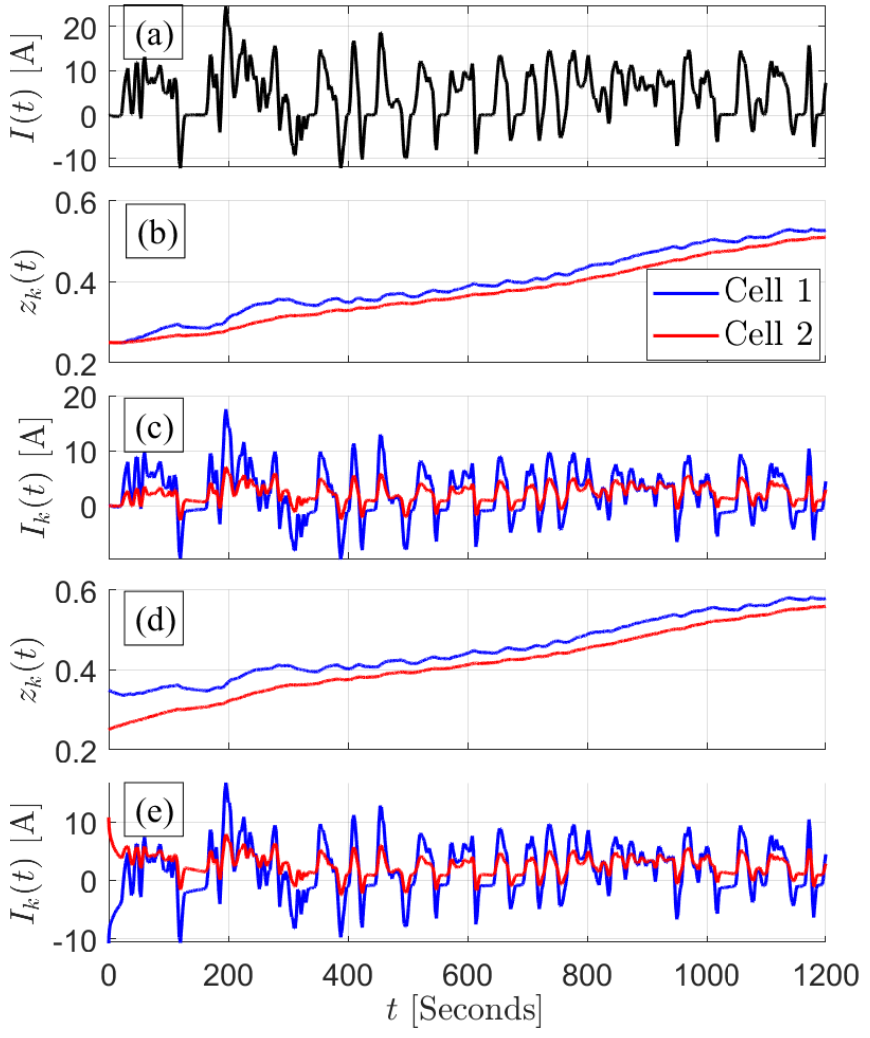

Fig. 2. Simulation results of two cells in parallel. In (b)-(c), cells are initialized at the same SOC, and the total current distributes unevenly due to parameter heterogeneity. In (d)-(e), the initial cell SOCs are distinct. The total current again distributes unevenly due to both parameter and initialization heterogeneity.

even though the applied total current is small (around zero) initially, Cell 1 takes large negative current (around -10 A) and Cell 2 positions itself at a large positive current (around $+10 \mathrm{~A})$. This occurs because $z_{1}(0)$ is initialized higher, and even though the $z$ values for two cells follow a similar trend, they do not synchronize - a bias persists. These results demonstrate how heterogeneous parameters can yield nonuniform state trajectories in parallel connected cells. This issue is accentuated when the cells differ in SOC, which occurs in the absence of SOC balancing. Since conventional BMSs do not monitor the local current of each parallel cell, some cells might be operating outside their safe operating region. Therefore, it is significantly valuable to monitor the local currents and SOCs caused by cell heterogeneity to ensure safe battery pack operation.

\section{OBSERVABILITY ANALYSIS}

In this section, we mathematically analyze the observability of the nonlinear descriptor system with the input-output setup via Lie algebra for the nonlinear systems resulting from a reduced descriptor system. That is, we analyze the system that results from eliminating the algebraic states through substitution. System (7)-(8) can be divided into differential 
and algebraic states with an explicit input current, i.e.

$$
\begin{gathered}
{\left[\begin{array}{cc}
I_{2 n \times 2 n} & 0 \\
0 & 0
\end{array}\right] \dot{w}(t)=\left[\begin{array}{ll}
A_{11} & A_{12} \\
A_{21} & A_{22}
\end{array}\right] w(t)+\left[\begin{array}{c}
0 \\
\theta_{I}
\end{array}\right] I(t)+\left[\begin{array}{c}
\theta_{x}(w) \\
\theta_{O C V}(w)
\end{array}\right],} \\
y(t)=\left[\begin{array}{ll}
H_{1} & H_{2}
\end{array}\right] w(t)+\phi(w) .
\end{gathered}
$$

where $\theta_{I}=[0, \cdots,-1]^{\top} \in \mathbb{R}^{n}$ and $\theta_{O C V}(w)=\theta_{u}(w)-\theta_{I} I(t)$. Eq. (18) can be solved for the algebraic state, resulting in the following transformation

$$
u=-A_{22}^{-1}\left(A_{21} x(t)+\theta_{I} I(t)+\theta_{O C V}(w)\right)
$$

where matrix $A_{22}$ in (13) is non-singular. Notice that (20) is an explicit solution for the algebraic state $u(t)$, i.e. the $w$-dependent functions $\theta_{O C V}(w)$ and $\phi(w)$ are now only dependent on the dynamic state $x(t)$. With an abuse of notation, these functions are denoted as $\theta_{O C V}(x)$ and $\phi(x)$ in the remainder of this section. For its part, $\theta_{x}(w)=\mathbf{0}_{2 n \times 1}=\theta_{x}$.

Substituting (20) back into the differential part of the state equation (18) and the output equation (19) yields the following nonlinear (control affine) reduced model

$$
\begin{aligned}
& \dot{x}(t)=f(x(t))+g(x(t)) I(t), \\
& \bar{y}(t)=h(x(t)),
\end{aligned}
$$

with

$$
\begin{aligned}
& f(x)=\left(A_{11}-A_{12} A_{22}^{-1} A_{21}\right) x(t)-A_{12} A_{22}^{-1} \theta_{O C V}(x)+\theta_{x}, \\
& g(x)=-A_{12} A_{22}^{-1} \theta_{I}, \\
& h(x)=\left(H_{1}-H_{2} A_{22}^{-1} A_{21}\right) x(t)-H_{2} A_{22}^{-1} \theta_{O C V}(x)+\phi(x),
\end{aligned}
$$

where the output $\bar{y}(t)=y(t)-H_{2} A_{22}^{-1} \theta_{I} I(t)$.

Let us now introduce the notion of local observability [23] for system (21)-(22).

Theorem 1 ([23]): The system (21)-(22) is locally observable around $x_{0} \in X$ if there exists $n$ linearly independent row vectors in the set

$$
\left(\mathrm{d} L_{z_{s}} L_{z_{s-1}} \ldots L_{z_{1}} h_{j}\right)\left(x_{0}\right)
$$

where $L_{z_{s}} h_{j}$ are Lie derivatives of $h_{j}$ with respect to $z_{s}$ and $\mathrm{d} h_{j}$ is the gradient of $h_{j}$ to be defined below, $s \geq 0$ and $z_{i} \in\{f, g\}$, with $j=1, \ldots, p$ ( $p$ is the number of outputs) and $i=1, \ldots, s$ (for $s=0$, the set comprises $\mathrm{d} h_{j}\left(x_{0}\right)$ ).

In Theorem 1, the gradient of $h_{j}$ and Lie derivatives of $h_{j}$ with respect to function $f$ are given by

$$
\mathrm{d} h_{j}=\left[\begin{array}{llll}
\frac{\partial h_{j}}{\partial x_{1}} & \frac{\partial h_{j}}{\partial x_{2}} & \cdots & \frac{\partial h_{j}}{\partial x_{n}}
\end{array}\right], \quad L_{f} h_{j}=\left\langle\mathrm{d} h_{j}, f\right\rangle,
$$

and similarly for function $g$. The 0 th order Lie derivative $L_{f}^{0} h_{j}$ is defined as $h_{j}$ whereas the 2 nd order Lie derivative takes the form $L_{f}^{2} h_{j}=L_{f} L_{f} h_{j}$.

According to Theorem 1, the observability rank condition can be derived. Define the observability matrix as

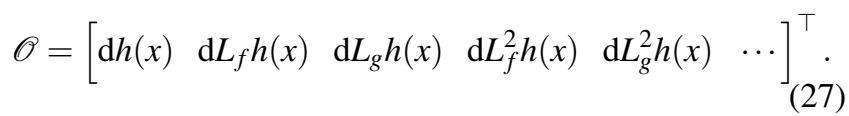

Then the model (21)-(22) is locally observable around $x_{0}$ if $\operatorname{rank}(\mathscr{O})=2 n$. Note first that the observability matrix $\mathscr{O}$ is not bounded from above. Secondly, this matrix depends on states $x_{k}=\left[z_{k}, V_{c, k}\right]^{\top}$, functions $O C V_{k}\left(z_{k}\right)$ as well as parameters $\theta_{k}=\left[R_{1, k}, \tau_{k}, C_{k}, Q_{k}\right]^{\top}$, where $\tau_{k}=-1 /\left(R_{2, k} C_{k}\right)$.

To keep the observability analysis tractable, we consider two cells in parallel, i.e. $k \in\{1,2\}$. From the observability rank condition, we conclude: (i) the system is locally observable at $x_{0}$ if cells have different parameter values $\theta_{k}$, and (ii) observability conditions are not fulfilled if the cells are completely equivalent, i.e. all parameter values are uniform across the cells. In the second case, the cells are presumably indistinguishable and a single cell model can be utilized to represent the parallel connection. In between these two extremes, the observability conditions cannot be verified if any of the following conditions hold:

1) the parameters $\tau_{1}=\tau_{2}$ AND $R_{1,1} Q_{1}=R_{1,2} Q_{2}$ AND $R_{1,1} C_{1}=R_{1,2} C_{2}$;

2) the functions $O C V\left(z_{1}\right)=O C V\left(z_{2}\right)$ AND $\operatorname{dOCV}\left(z_{1}\right) / d z_{1}=\operatorname{dOCV}\left(z_{2}\right) / d z_{2}$

$3)$ at least one of the $l$-th OCV derivatives satisfy $d^{l} O C V\left(z_{j}\right) / d z_{j}^{l}=0$, for the $j$-th cell and $l=1, \ldots, \infty$.

Note that a common approach to study observability of a single ECM is to linearize the model, as done in e.g. [24], for the case of two battery cells in parallel. By doing so, the ECM is observable if $\mathrm{d} O C V / \mathrm{d} z \neq 0$. This condition on the first OCV derivative is conservative, as it was found in [25] through the local observability analysis of a single cell. In other words, the condition $\mathrm{d} O C V / \mathrm{d} z \neq 0$ is sufficient for observability, but not necessary. More general conditions exist. The detailed analysis in [25] showed that an OCV derivative must be different than zero to guarantee local observability, but it does not need to be the first derivative. This fact is also verified here for two ECMs in parallel.

A similar observability analysis was also carried out in [3] considering cells in series. However, the observability matrix for a series string differs from that of a parallel arrangement. Namely, in the series arrangement, each cell's parameters/states appear in a column of $\mathscr{O}$. This is not the case for a parallel topology, whose parameters/states are scattered all over the different entries in $\mathscr{O}$. Therefore, parameters/states of one cell influence the local observability of the neighbouring cell in a parallel arrangement.

When compared to observability analysis for linearized descriptor systems, the local observability analysis of the nonlinear system using Theorem 1 is less conservative [23] and more informative. The latter aspect relies on the fact that the observability matrix $\mathscr{O}$ explicitly depends on model parameters, and it can be analytically obtained through e.g. symbolic software. On the other hand, an arbitrary number of cells should be considered in order to generalize the observability conditions found here for the two cells case.

\section{Design of State Observers}

The following observer with linear output error injection is proposed for the plant model (7)-(8):

$$
\begin{aligned}
E \dot{\hat{w}} & =A \hat{w}+\theta(\hat{w})+K(y-H \hat{w}-\phi(\hat{w})), \\
\hat{y} & =H \hat{w}+\phi(\hat{w}),
\end{aligned}
$$


where $K \in \mathbb{R}^{3 n}$ is the observer gain to be designed and $\hat{w}$ is the estimate of $w$. The following theorem using results from [16], [17] establishes estimation error convergence.

Theorem 2: Consider the plant model dynamics (7)-(8), and suppose the matrix $\left[\begin{array}{ll}A_{22} & H_{u}\end{array}\right]^{\top}$ has rank $n$. Let

$$
G=(A-K H)=\left[\begin{array}{ll}
G_{11} & G_{12} \\
G_{21} & G_{22}
\end{array}\right],
$$

and define the matrix

$$
\widetilde{G}=\left(G_{11}-G_{12} G_{22}^{-1} G_{21}\right) .
$$

Suppose the function

$$
f(w)=\theta_{x}(w)-G_{12} G_{22}^{-1} \theta_{u}(w)+\left(G_{12} G_{22}^{-1} K_{u}-K_{x}\right) \phi(w),
$$

is Lipschitz continuous with respect to $x$, i.e.,

$$
\left\|f\left(x_{1}, u\right)-f\left(x_{2}, u\right)\right\| \leq \gamma\left\|x_{1}-x_{2}\right\|,
$$

where $\gamma \in \mathbb{R}$ is the Lipschitz constant. If the observer gain $K$ is chosen to ensure that $\widetilde{G}$ is stable, and

$$
\min _{\omega \in \mathbb{R}^{+}} \lambda_{\min }\left(A-K H-j \omega I_{3 n \times 3 n}\right)>\gamma,
$$

then the zero equilibrium of the dynamics of estimation error $e(t)=w(t)-\hat{w}(t)$ is asymptotically convergent to zero.

Proof: Let the state estimation error $e=\left[\begin{array}{ll}e_{x} & e_{u}\end{array}\right]^{\top}$, with $e_{x}=x-\hat{x}$ being the estimation error for the differential states and $e_{u}=u-\hat{u}$ the estimation error for the algebraic states. Then the dynamics for $e$ can be written as

$$
\begin{aligned}
{\left[\begin{array}{cc}
I_{2 n \times 2 n} & \mathbf{0} \\
\mathbf{0} & \mathbf{0}
\end{array}\right]\left[\begin{array}{c}
\dot{e}_{x} \\
\dot{e}_{u}
\end{array}\right]=} & {\left[\begin{array}{ll}
G_{11} & G_{12} \\
G_{21} & G_{22}
\end{array}\right]\left[\begin{array}{c}
e_{x} \\
e_{u}
\end{array}\right]+\left[\begin{array}{l}
\theta_{x}(w)-\theta_{x}(\hat{w}) \\
\theta_{u}(w)-\theta_{u}(\hat{w})
\end{array}\right] } \\
& -\left[\begin{array}{l}
K_{x} \\
K_{u}
\end{array}\right][\phi(w)-\phi(\hat{w})]
\end{aligned}
$$

We highlight that $G_{22}$ can be non-singular (invertible) if the linear part of (7) is impulse observable, i.e. the matrix $\left[\begin{array}{ll}A_{22} & H_{u}\end{array}\right]^{\top}$ has rank $n$ [17]. Then the estimation error system (35) is equivalently described by

$$
\begin{aligned}
\dot{e}_{x}= & \left(G_{11}-G_{12} G_{22}^{-1} G_{21}\right) e_{x}+\left[\theta_{x}(w)-G_{12} G_{22}^{-1} \theta_{u}(w)\right] \\
& -\left[\theta_{x}(\hat{w})-G_{12} G_{22}^{-1} \theta_{u}(\hat{w})\right]+\left(G_{12} G_{22}^{-1} K_{u}-K_{x}\right) \phi(w) \\
& -\left(G_{12} G_{22}^{-1} K_{u}-K_{x}\right) \phi(\hat{w}) \\
= & \widetilde{G} e_{x}+f(w)-f(\hat{w}),
\end{aligned}
$$

along with the algebraic equation

$$
\begin{aligned}
e_{u}= & -G_{22}^{-1} G_{21} e_{x}-G_{22}^{-1}\left[\theta_{u}(w)-\theta_{u}(\hat{w})\right] \\
& +G_{22}^{-1} K_{u}[\phi(w)-\phi(\hat{w})] .
\end{aligned}
$$

Consider the following Lyapunov function for the error system (36), corresponding to the differential states $e_{x}$,

$$
W(t)=\frac{1}{2} e_{x}^{\top} P e_{x} .
$$
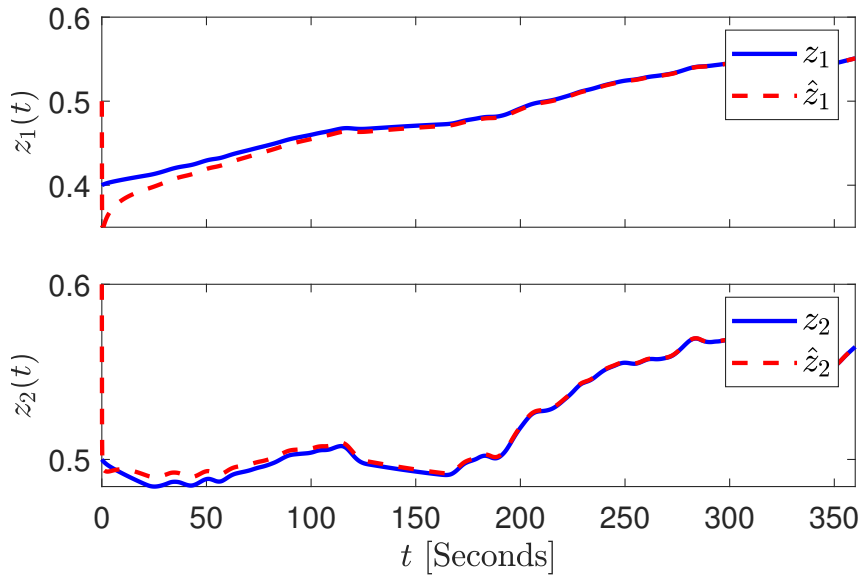

Fig. 3. The estimation performance for SOCs of a two-cell parallel configuration. The results verify the asymptotic convergence.
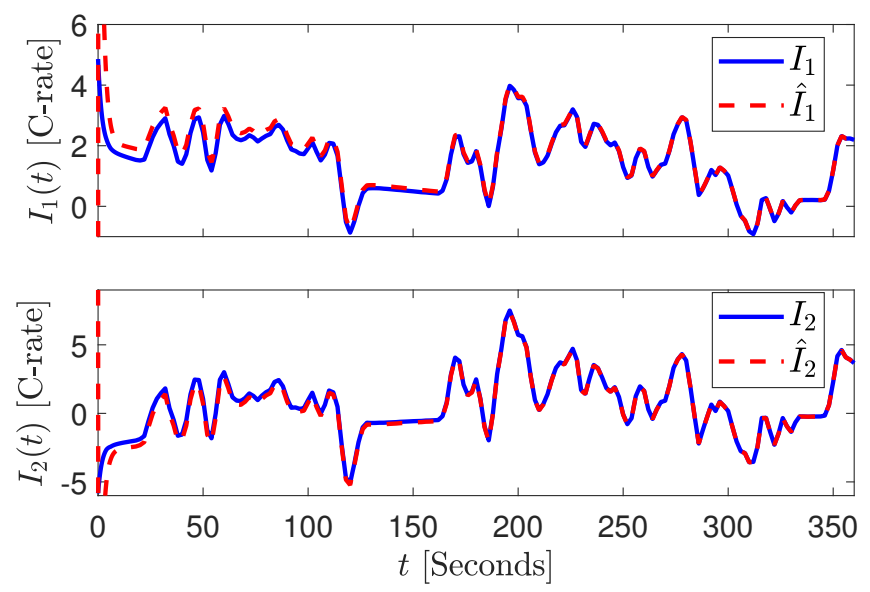

Fig. 4. The estimation performance for local currents of a two-cell parallel configuration. The results verify the asymptotic convergence.

The time derivative of $W(t)$ is given by

$$
\begin{aligned}
\dot{W} & =\frac{1}{2} \dot{e}_{x}^{\top} P e_{x}+\frac{1}{2} e_{x}^{\top} P \dot{e}_{x} \\
& =\frac{1}{2} e_{x}^{\top}\left(\widetilde{G}^{\top} P+P \widetilde{G}\right) e_{x}+e_{x}^{\top} P[f(w)-f(\hat{w})] \\
& \leq \frac{1}{2} e_{x}^{\top}\left(\widetilde{G}^{\top} P+P \widetilde{G}\right) e_{x}+\left\|P e_{x}\right\|[\|f(w)-f(\hat{w})\|] \\
& \leq \frac{1}{2} e_{x}^{\top}\left(\widetilde{G}^{\top} P+P \widetilde{G}\right) e_{x}+\gamma\left\|P e_{x}\right\|\left\|e_{x}\right\| \\
& \leq \frac{1}{2} e_{x}^{\top}\left[\widetilde{G}^{\top} P+P \widetilde{G}+\gamma^{2} P P+I\right] e_{x},
\end{aligned}
$$

where the inequality

$$
2 \gamma\left\|P e_{x}\right\|\left\|e_{x}\right\| \leq \gamma^{2} e_{x}^{\top} P P e_{x}+e_{x}^{\top} e_{x}
$$

has been utilized. According to Theorem 2 in [16], the estimation error $e_{x}$ is asymptotically stable if the conditions of Theorem 2 hold. Therefore, when $t \rightarrow \infty,\left[\theta_{u}(w)-\theta_{u}(\hat{w})\right] \rightarrow$ $\mathbf{0}_{n \times 1}$, and $[\phi(w)-\phi(\hat{w})] \rightarrow 0$. Hence, the estimation error $e_{u}$ for the algebraic states also converge to zero asymptotically.

\section{Simulation Study}

A simulation study with $n=2$ NMC cells in parallel is conducted to evaluate the performance of the proposed 
TABLE I

Model Parameters in Simulation Study

\begin{tabular}{cccc}
\hline \hline & Cell 1 & Cell 2 & Units \\
\hline$R_{1, k}$ & 0.0035 & 0.0015 & {$[\Omega]$} \\
$R_{2, k}$ & 0.004 & 0.0025 & {$[\Omega]$} \\
$C_{k}$ & 1000 & 2000 & {$[\mathrm{~F}]$} \\
$Q_{k}$ & 2.4 & 2.0 & {$[\mathrm{Ah}]$} \\
$z_{0}$ & 0.4 & 0.5 & {$[-]$} \\
$O C V(z)$ & $-0.79 \exp (-30 z)+3.52 \exp (-0.0092 z)+0.68 z^{2}$ & {$[\mathrm{~V}]$} \\
\hline \hline
\end{tabular}

estimation scheme. Without loss of generality, a pair of cells is preferred over a larger block to facilitate the presentation of results. We consider the situation in which the cells may differ in their initial SOCs and model parameters, but subject to the same SOC-OCV relationship. This setup guarantees local observability based on the analysis in Section IV-A. The model parameters and initial SOCs are shown in Table I. Under these circumstances, the state vector is given by $w=\left[\begin{array}{llllll}z_{1} & V_{c, 1} & z_{2} & V_{c, 2} & I_{1} & I_{2}\end{array}\right]^{\top} \in \mathbb{R}^{6}$. The total applied current comes from a UDDS drive cycle in Fig. 2(a) with appropriate scaling, where $I>0$ denotes charging. The observer in (28)(29) estimates the individual cell SOCs and the local currents by using only the voltage and overall current measurements. The initial SOC errors between the plant model and the observer are $10 \%$. The observer gain is chosen to be $K=$ $\left[\begin{array}{lllll}-30 & -30 & -20 & 2 & 4\end{array}-20\right]^{\top}$, which satisfies Theorem 2 .

Figs. 3 and 4 demonstrate estimation performance, where the solid blue curves are the simulated states from the plant model and the dashed red curves are the estimated ones. Fig. 3 displays the estimates for the differential states (SOCs), whereas Fig. 4 portrays the estimates for the algebraic states (local currents). The observers are able to recover quickly (within approximately 100 seconds) from large initial estimation errors. These results confirm the asymptotic zero error convergence conclusions in Theorem 2.

\section{CONCLUSION}

A nonlinear descriptor system has been proposed to model parallel arrangements of lithium-ion battery cells, and a state observer for such systems has been developed. This modeling framework fits naturally with battery applications, given the interconnections arising from Kirchhoff's laws. The design procedure used to build the state observer from this model avoids linearization or canonical transformations, and it only relies on the assumption of Lipschitz nonlinearities. The resulting state observer benefits from considering the cell currents as algebraic states to be simultaneously estimated with the differential states. The effectiveness of the proposed estimation approach was demonstrated in simulation.

\section{ACKNOWLEDGEMENT}

Luis D. Couto would like to thank the Wiener-Anspach Foundation for its financial support.

\section{REFERENCES}

[1] N. A. Chaturvedi, R. Klein, J. Christensen, J. Ahmed, and A. Kojic, "Algorithms for advanced battery-management systems," IEEE Control systems magazine, vol. 30, no. 3, pp. 49-68, 2010.
[2] L. Zhong, C. Zhang, Y. He, and Z. Chen, "A method for the estimation of the battery pack state of charge based on in-pack cells uniformity analysis," Applied Energy, vol. 113, pp. 558-564, 2014.

[3] X. Lin, A. G. Stefanopoulou, Y. Li, and R. D. Anderson, "State of charge imbalance estimation for battery strings under reduced voltage sensing," IEEE Transactions on Control Systems Technology, vol. 23 no. 3, pp. 1052-1062, 2015.

[4] S. Castano, L. Gauchia, E. Voncila, and J. Sanz, "Dynamical modeling procedure of a Li-ion battery pack suitable for real-time applications," Energy Conversion and Management, vol. 92, pp. 396-405, 2015.

[5] Y. Hua, A. Cordoba-Arenas, N. Warner, and G. Rizzoni, "A multi timescale state-of-charge and state-of-health estimation framework using nonlinear predictive filter for lithium-ion battery pack with passive balance control," Journal of Power Sources, vol. 280, pp. 293-312, 2015

[6] Y. Zheng, M. Ouyang, L. Lu, J. Li, X. Han, L. Xu, H. Ma, T. A. Dollmeyer, and V. Freyermuth, "Cell state-of-charge inconsistency estimation for $\mathrm{LiFePO}_{4}$ battery pack in hybrid electric vehicles using mean-difference model," Applied Energy, vol. 111, pp. 571-580, 2013.

[7] X. Zhang, Y. Wang, D. Yang, and Z. Chen, "An on-line estimation of battery pack parameters and state-of-charge using dual filters based on pack model," Energy, vol. 115, pp. 219-229, 2016.

[8] X. Hu, F. Sun, and Y. Zou, "Estimation of state of charge of a lithiumion battery pack for electric vehicles using an adaptive Luenberger observer," Energies, vol. 3, no. 9, pp. 1586-1603, 2010.

[9] T. Bruen and J. Marco, "Modelling and experimental evaluation of parallel connected lithium ion cells for an electric vehicle battery system," Journal of Power Sources, vol. 310, pp. 91-101, 2016.

[10] G.-R. Duan, Analysis and design of descriptor linear systems, vol. 23. Springer Science \& Business Media, 2010.

[11] R. Nikoukhah, A. S. Willsky, and B. C. Levy, "Kalman filtering and Riccati equations for descriptor systems," IEEE Transactions on Automatic Control, vol. 37, no. 9, pp. 1325-1342, 1992.

[12] M. Darouach and M. Boutayeb, "Design of observers for descriptor systems," IEEE Transactions on Automatic Control, vol. 40, no. 7, pp. 1323-1327, 1995.

[13] M. Boutayeb and M. Darouach, "Observers design for nonlinear descriptor systems," in 34th IEEE Conference on Decision and Control, vol. 3, pp. 2369-2374 vol.3, 1995.

[14] G. Zimmer and J. Meier, "On observing nonlinear descriptor systems,' Systems \& Control Letters, vol. 32, no. 1, pp. 43-48, 1997.

[15] J. Åslund and E. Frisk, "An observer for non-linear differentialalgebraic systems," Automatica, vol. 42, no. 6, pp. 959-965, 2006.

[16] R. Rajamani, "Observers for lipschitz nonlinear systems," IEEE transactions on Automatic Control, vol. 43, no. 3, pp. 397-401, 1998.

[17] S. Kaprielian and J. Turi, "An observer for a nonlinear descriptor system," in 31st IEEE Conference on Decision and Control, pp. 975 976, 1992.

[18] L. Guoping and D. W. C. Ho, "Full-order and reduced-order observers for Lipschitz descriptor systems: the unified LMI approach," IEEE Transactions on Circuits and Systems II: Express Briefs, vol. 53, no. 7 , pp. 563-567, 2006.

[19] M. Darouach and L. Boutat-Baddas, "Observers for a class of nonlinear singular systems," IEEE Transactions on Automatic Control, vol. 53, no. 11, pp. 2627-2633, 2008.

[20] D. N. Shields, "Observer design and detection for nonlinear descriptor systems," International Journal of Control, vol. 67, no. 2, pp. 153-168, 1997.

[21] J. S. Albuquerque and L. T. Biegler, "Decomposition algorithms for on-line estimation with nonlinear DAE models," Computers \& chemical engineering, vol. 21, no. 3, pp. 283-299, 1997.

[22] V. M. Becerra, P. D. Roberts, and G. W. Griffiths, "Applying the extended Kalman filter to systems described by nonlinear differentialalgebraic equations," Control Engineering Practice, vol. 9, no. 3, pp. 267-281, 2001.

[23] M. Vidyasagar, Nonlinear Systems Analysis, vol. 42. Philadelphia, PA, USA: SIAM, 2002.

[24] M. Rausch, S. Streif, C. Pankiewitz, and R. Findeisen, "Nonlinear observability and identifiability of single cells in battery packs," in 2013 IEEE International Conference on Control Applications, pp. 401-406, 2013.

[25] S. Zhao, S. R. Duncan, and D. A. Howey, "Observability analysis and state estimation of lithium-ion batteries in the presence of sensor biases," IEEE Transactions on Control Systems Technology, vol. 25, no. 1, pp. 326-333, 2017. 